\title{
VLT FORS2 optical imaging and spectroscopy of nine luminous type 2 AGN at $0.3<z<0.6-$ I. Ionized gas nebulae
}

\author{
A. Humphrey, ${ }^{1 \star}$ M. Villar-Martín, ${ }^{2,3}$ C. Ramos Almeida, ${ }^{4}$ C. N. Tadhunter, ${ }^{5}$ \\ S. Arribas, ${ }^{2,3}$ P. S. Bessiere ${ }^{6}$ and A. Cabrera-Lavers ${ }^{4}$ \\ ${ }^{1}$ Instituto de Astrofísica e Ciências do Espaço, Universidade do Porto, CAUP, Rua das Estrelas, P-T4150-762 Porto, Portugal \\ ${ }^{2}$ Centro de Astrobiología (INTA-CSIC), Carretera de Ajalvir, km 4, E-28850 Torrejón de Ardoz, Madrid, Spain \\ ${ }^{3}$ Astro-UAM, UAM, Unidad Asociada CSIC, Facultad de Ciencias, Campus de Cantoblanco, E-28049 Madrid, Spain \\ ${ }^{4}$ Instituto de Astrofísica de Canarias (IAC), Vía Láctea s/n, E-38205 La Laguna, Tenerife, Spain \\ ${ }^{5}$ Department of Physics and Astronomy, University of Sheffield, Sheffield S3 7RH, UK \\ ${ }^{6}$ Universidad de Concepción, Departamento de Astronomía, Casilla 160-C, Concepción, Chile
}

Accepted 2015 September 17. Received 2015 September 17; in original form 2015 July 30

\begin{abstract}
We present optical imaging and long slit spectroscopic observations of nine luminous type 2 active galactic nuclei (AGN) within the redshift range $0.3<z<0.6$ based on Very Large Telescope Focal Reducer and Low Dispersion Spectrograph (VLT FORS2) data. Most objects (6/9) are high luminosity Seyfert 2, and three are type 2 quasars (QSO2), with our sample extending to lower luminosity than previous works. Seven out of nine objects (78 per cent) show morphological evidence for interactions or mergers in the form of disturbed morphologies and/or peculiar features such as tidal tails, amorphous haloes, or compact emission line knots. The detection rate of morphological evidence for interaction is consistent with those found during previous studies of QSO2 at similar $z$, suggesting that the merger rate is independent of AGN power at the high end of the AGN luminosity function. We find the emission line flux spatial profiles are often dominated by the often spatially unresolved central source. In addition, all but one of our samples is associated with much fainter, extended line emission. We find these extended emission line structures have a variety of origins and ionization mechanisms: star-forming companions, tidal features, or extended ionized nebulae. AGN related processes dominate the excitation of the nuclear gas. Stellar photoionization sometimes plays a role in extended structures often related to mergers/interactions.
\end{abstract}

Key words: galaxies: active-galaxies: evolution-galaxies: interactions-galaxies: ISMgalaxies: nuclei-quasars: emission lines.

\section{INTRODUCTION}

The coevolution and interplay of galaxies with their nuclear black hole is a topic of key importance for understanding galaxy evolution. Negative feedback from an active galactic nucleus (hereinafter AGN) may impact on the evolution of the stellar and gaseous components of the host galaxy, by heating or expelling cold gas that may otherwise have formed stars or fed the AGN, potentially hindering mass assembly activity. Feedback such as this has been invoked to explain the deviation of the observed galaxy stellar mass function from the theoretical function (White \& Frenk 1991; Puchwein \& Springel 2013), to account the observed correlation between the black hole mass and the stellar mass of the stellar spheroidal component of the host galaxy (Magorrian et al. 1998), and to facilitate the transformation of dusty, obscured galaxies to unobscured,

${ }^{\star}$ E-mail: andrew.humphrey@ astro.up.pt optical galaxies (e.g. Sanders et al. 1988; Bessiere et al. 2014). Although some negative feedback clearly does take place in galaxies as they undergo a phase of AGN activity, the dominant mechanism and strength of this feedback remains a matter of debate in the literature. Positive feedback, where star formation is triggered or enhanced due to AGN-related processes, has also been suggested to take place under some circumstances (Rees 1989; Silk 2013), with a few tentative detections in the literature (Croft et al. 2006; Stroe et al. 2014).

Triggering is another key issue for understanding AGN activity and its relationship with the evolution of the host galaxy. Gas-rich major mergers are thought able to trigger powerful nuclear activity, insofar as they provide a mechanism to displace large quantities of cold gas into the central few kiloparsecs (kpc) of one (or more) of the merging galaxies (e.g. Heckman et al. 1986), and there is a growing body of evidence to support this idea (e.g. Ramos Almeida et al. 2011, 2012; Tadhunter et al. 2011; Bessiere et al. 2012, 2014). 
Radio quiet, QSO2 are a potential goldmine of information to improve our understanding of several important aspects of the evolution of massive galaxies, including the triggering and impact of AGN activity therein. One of their main advantages is that the fortuitous obscuration by an optically thick structure of the highly luminous central engine, which can otherwise outshine the entire stellar content of the host galaxy, affords a cleaner picture of the galaxy. Moreover, compared to their radio-loud cousins (powerful radio galaxies), the space density of radio-quiet QSO2 is roughly an order of magnitude higher (Reyes et al. 2008), making them far more representative of powerful active galaxies. In addition, the absence of powerful radio jets removes substantial ambiguity about whether or not observed properties of the host galaxy are induced by the radio jets, which often impacts studies of radio loud quasars and radio galaxies. However, their lack of powerful radio emission or highly luminous optical emission meant that, even after their existence was hypothesized during efforts to unify the seemingly disparate varieties of active galaxy radio quiet, QSO2 remained relatively elusive for decades (see e.g. Halpern, Turner \& George 1999).

It was not until the advent of the Sloan Digital Sky Survey (SDSS) that type 2 radio quiet quasars were identified in significant numbers. Using selection criteria designed to find galaxies that contain gas photoionized by a powerful but obscured AGN, Zakamska et al. (2003) were able to identify $\sim 300$ candidate QSO2 within the range $0.3<z<0.8$, of which 85 per cent are radio quiet (see also Reyes et al. 2008). Subsequent observations at various wavelengths confirmed that these galaxies are indeed QSO2, with unobscured luminosities that place them among the most luminous quasars at similar redshifts (Zakamska et al. 2004, 2006; Ptak et al. 2006; Lal \& Ho 2010). Complementary to the above, radio quiet QSO2 have now also been found in significant numbers by using data from infrared, X-ray and radio surveys to select heavily obscured AGN (e.g. Ohta et al. 1996; Norman et al. 2002; Martínez-Sansigre et al. 2005, 2006a,b), or by using redshifted ultraviolet emission lines (e.g. Ly $\alpha$ ) to select QSO2 at $z \gtrsim 2$ (Alexandroff et al. 2013). At the time of writing, almost 1000 optically selected QSO2 have now been identified (e.g. Reyes et al. 2008).

The bright optical emission lines that facilitate the identification of QSO2 at low to intermediate redshift also carry information about the properties of the gaseous component of the host galaxy. Spatially extended ( $\gtrsim 10 \mathrm{kpc}$ ) regions of warm $(T \sim 10000 \mathrm{~K})$ ionized gas are frequently detected (e.g. Humphrey et al. 2010; Villar-Martín et al. 2011a, hereinafter VM11a; Liu et al. 2013a, Liu et al.2013b; Harrison et al. 2014; McElroy et al. 2015). In most cases, the excitation of this gas is dominated by photoionization by the hard radiation field of the central AGN, with an occasional contribution from young stars (Villar-Martín et al. 2008) or, in at least one case, a contribution from shock ionization (Humphrey et al. 2010). Many of these quasars show evidence for outflows in their nuclear narrow line region (Humphrey et al. 2010; VillarMartín et al. 2011b, hereinafter VM11b, 2012; Liu et al. 2013b; see also e.g. Arribas et al. 2014). In addition, some intermediate redshift QSO2 also contain a large mass of molecular gas, as detected in $\mathrm{CO}$ line emission (Villar-Martín et al. 2013a, Villar-Martín et al. 2013b; Rodríguez et al. 2014).

The host galaxies of the majority of intermediate redshift QSO2 show elliptical morphologies, with around half showing morphological disturbances identifiable with galaxy merger or interaction (Bessiere et al. 2012; Villar-Martín et al. 2012). Although their stellar populations are dominated by old stars, young or poststarburst populations are also prevalent (e.g. Bian 2007; Bessiere et al. in preparation), suggesting roughly synchronous triggering of the AGN and starburst activity during a merger event, albeit with rather different delays from the starburst to AGN triggering from quasar to quasar (see, e.g. Bessiere et al. 2014). Arguments from environmental clustering amplitudes suggest that all massive elliptical galaxies go through a short-lived phase as a radio-quiet quasar (Ramos Almeida et al. 2013).

In 2008 we started an observational programme with the Very Large Telescope (VLT) based on optical imaging and spectroscopy of QSO2, with the goal of investigating, quantifying and characterizing (1) the existence of ionized outflows, (2) the incidence of interactions/mergers and (3) the existence, properties and origin of extended ionized structures in the most powerful radio quiet type 2 active galaxies. In this paper, we extend the work published in four earlier papers (Villar-Martín et al. 2010, 2012; VM11a; VM11b), focusing now on a somewhat less luminous sample. Here we concentrate on the optical morphology and merger status of the quasar host galaxies, and the spatial distribution and excitation of narrowline emitting gas therein. In a future paper (Villar-Martín et al. in preparation; Paper II) we will present a detailed kinematic analysis and discuss the properties of ionized gas outflows.

The paper is organized as follows. The sample is described in Section 2. The observations and data reduction are explained in Section 3. Analysis and results are presented in Section 4 both for the individual objects. The overall results are discussed in Section 5 and the conclusions are presented in Section 6.

We adopt $H_{0}=71 \mathrm{~km} \mathrm{~s}^{-1} \mathrm{Mpc}^{-1}, \Omega_{\Lambda}=0.73$ and $\Omega_{\mathrm{m}}=0.27$. At the redshifts of our sample, this gives an arcsec to kpc conversion ranging from 4.47 to $6.52 \mathrm{kpc} \operatorname{arcsec}^{-1}$.

\section{THE SAMPLE}

The sample consists of nine luminous type 2 AGN selected from the SDSS sample of high luminosity type 2 AGN selected by Reyes et al. (2008). They are objects with narrow $\left(<2000 \mathrm{~km} \mathrm{~s}^{-1}\right)$ emission lines without underlying broad components for the recombination lines suggestive of a broad-line region (BLR), and with line ratios characteristic of non-stellar ionizing radiation. Some basic information is presented in Table 1 . All objects have redshift $z \sim 0.3-0.6$ such that there was an adequate narrow- or intermediate-band VLT Focal Reducer and Low Dispersion Spectrograph (FORS2) filter containing one of the strongest emission lines in the optical spectrum ([O II] $\lambda 3727$ or [O III] $\lambda 5007)$. The [O III] luminosities are in the range $l_{\mathrm{O} 3}=\log \frac{L_{[\mathrm{Om}}}{\mathrm{L}_{\odot}}=7.8-8.8$ (luminosities taken from the Vizier online catalogue of Reyes et al. 2008). We selected our targets to have large emission line equivalent widths.

For completeness, in Table 1 we also give the flux density of each target at $1.4 \mathrm{GHz}$, as measured by the Faint Images of the Radio Sky at Twenty-Centimeters survey (FIRST; Becker, White \& Helfand 1995) and, where available, as measured by the NRAO VLA Sky Survey (NVSS; Condon et al. 1998). Issues such as the radio-loudness of the targets will be explored in Paper II.

The sample is similar to that studied by VM11a and VM11b. The main difference is that here we focus on somewhat less luminous objects. Our previous sample had a range of [O III] luminosities with median value corresponding to $l_{\mathrm{O} 3}=8.84$, while the current sample has 8.27. Zakamska et al. (2003) and Reyes et al. (2008) used slightly different criteria to select SDSS QSO2 based on their spectroscopic properties, imposing $l_{\mathrm{O} 3}>8.5$ and $>8.3$ respectively. Although these numbers are only indicative, and there might be a gradual transition of properties with increasing luminosity, we 
Table 1. The sample of QSO2 $\left(l_{\mathrm{O} 3}=\log \left(\frac{\left.L_{[\mathrm{O}} \mathrm{ml}\right]}{\mathrm{L}_{\odot}}\right)>8.3\right)$ and $\mathrm{HSy} 2\left(l_{\mathrm{O} 3}<8.3\right)$.

\begin{tabular}{|c|c|c|c|c|c|c|c|}
\hline Target & Short name & $z$ & $\log \left(\frac{L_{\left[\mathrm{O}_{\mathrm{III}}\right]}}{\mathrm{L}_{\odot}}\right)$ & $\begin{array}{l}\text { SDSS } \\
\text { (g mag) }\end{array}$ & $\begin{array}{c}\text { FIRST } S_{1.4 \mathrm{GHz}} \\
(\mathrm{mJy})\end{array}$ & $\begin{array}{c}\text { NVSS } S_{1.4 \mathrm{GHz}} \\
(\mathrm{mJy})\end{array}$ & Class \\
\hline SDSS J090307.83+021152.2 & SDSS J0903+02 & 0.329 & 8.79 & 19.5 & $22.5 \pm 0.1$ & $24.2 \pm 0.8$ & QSO2 \\
\hline SDSS J092318.06+010144.8 & SDSS J0923+01 & 0.386 & 8.78 & 20.4 & $1.0 \pm 0.2$ & $2.3 \pm 0.5$ & QSO2 \\
\hline SDSS J095044.69+011127.2 & SDSS J0950+01 & 0.404 & 8.22 & 21.1 & $1.7 \pm 0.1$ & & HSy 2 \\
\hline SDSS J101403.49+024416.4 & SDSS J1014+02 & 0.573 & 8.29 & 22.6 & $2.4 \pm 0.1$ & & HSy 2 \\
\hline SDSS J101718.63+033108.2 & SDSS J1017+03 & 0.453 & 8.27 & 21.4 & $2.8 \pm 0.2$ & & HSy 2 \\
\hline SDSS J124749.79+015212.6 & SDSS J1247+01 & 0.427 & 8.23 & 21.2 & $8.0 \pm 0.1$ & $34.1 \pm 1.6$ & HSy2 \\
\hline SDSS J133633.65-003936.4 & SDSS J1336-00 & 0.416 & 8.64 & 20.7 & $\leq 0.5$ & & QSO2 \\
\hline SDSS J141611.77-023117.1 & SDSS J1416-02 & 0.305 & 8.03 & 21.2 & $1.4 \pm 0.1$ & & HSy 2 \\
\hline SDSS J145201.73+005040.2 & SDSS J1452+00 & 0.315 & 7.82 & 21.3 & $0.5 \pm 0.2$ & & HSy 2 \\
\hline
\end{tabular}

have divided our sample in QSO2 and high luminosity Seyfert 2 (hereinafter HSy2) adopting Reyes et al. (2008) criteria (i.e. a transition value of $l_{\mathrm{O} 3}=8.3$ ). The classification does not change if we use the criteria of Zakamska et al. (2003) instead. As a result, while the sample of VM11a and VM11b consisted of 12 QSO2 and two HSy2, the current sample contains three QSO2 and six HSy2.

\section{OBSERVATIONS AND DATA REDUCTION}

\subsection{VLT observations}

The VLT observations were made during the dark nights of 2011 April 25-28 at the Antu unit of the VLT, as part of the programme 087.B-0034. The FORS2 (Appenzeller et al. 1998) was used in long-slit spectroscopy and imaging modes. The seeing full width at half-maximum (FWHM) varied between $\sim 0.6$ and $\sim 1.1$ arcsec during the run (Table 2).

For each quasar the observing procedure involved first obtaining an image through the v_HIGH broad-band filter, and in most cases an image through an intermediate or narrow band filter chosen to

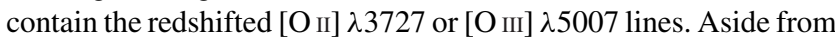
the valuable scientific information they provide, the images were used during the observing run to identify extended structures and possible companion objects, the presence and positions of which motivated our choice of slit position angle during the subsequent long-slit spectroscopic exposures.

The spectroscopic observations used one of two grisms. The 600RI grism gave a useful wavelength range of $\sim 5000-8000 \AA$, and a spectral resolution of $5.2 \pm 0.2 \AA$ (FWHM) when using the 1.0 arcsec slit, or $7.2 \pm 0.2 \AA$ when using the 1.3 arcsec slit. The $300 \mathrm{I}$ grism provided a useful range of $\sim 6000-10000 \AA$, and a resolution of $9.6 \pm 0.3 \AA$ with the 1.0 arcsec slit. The spatial pixel scale is 0.25 arcsec pixel $^{-1}$. The data were processed and calibrated using IRAF to apply standard data reduction techniques (see Villar-Martín et al. 2012 for details).

A total of ten luminous type 2 AGN at $0.3<z<0.6$ were observed during this VLT programme; the results for one of these (SDSS J143027.66-005614.8) have been published in a separate paper (Villar-Martín et al. 2012).

\subsubsection{Seeing and slit effects}

One of the main objectives of this observational programme is to examine the spatial properties of the narrow emission line gas of our sample (this paper), and to determine whether extended ionized outflows are present (Paper II). For this, a careful characterization of the seeing size (FWHM) and shape and its uncertainties is crucial.
The seeing was very variable during the VLT observations (FWHM in the range $\sim 0.6-1.3$ arcsec). To account for the uncertainties, we quote in Table 2 the seeing size measured from the broad-band and narrow/intermediate band images using several stars in the field (column 8) and the average FWHM seeing conditions calculated over the exposure time as measured by the differential image motion monitor (DIMM) station (column 9). The dispersion in these values will be carefully taken into account, especially when it could have an impact on our conclusions regarding the spatial extension of a given object.

In addition, we have reconstructed the spatial profile of the seeing disc along the slit, using a non-saturated star in images taken immediately before or after the spectroscopic observation of the science target. Because our study of the spatial extension of the ionized gas will be based primarily on [O III] $\lambda 5007$, whenever possible the narrow or intermediate band image containing this line was used, in order to minimize the effects of the wavelength dependence of the seeing profile. In the absence of either a narrow band or intermediate band image, the broad-band image was instead used. In all cases, the selected star was sufficiently bright to detect and trace adequately the faint wings of the seeing profile. The stellar flux was extracted from apertures centred on the stellar centroid and mimicking the slit: 4 (1.0 arcsec) or 5 pixels ( 1.3 arcsec $)$ wide depending on the slit used for the science target's spectroscopic observation. Finally, the sky background was removed from the stellar spatial profiles.

For some of our science targets, the $\left[\mathrm{O}_{\mathrm{II}}\right] \lambda 3727$ line was within the observed spectral range, and should provide some useful information to complement that to be obtained using [O III], provided the wavelength dependence of the seeing profile is understood and taken into account. To this end, we have used standard star spectra, obtained during the observing run (albeit at different air masses), and studied the star's seeing point spread function as a function of wavelength. We find that the FWHM of the seeing profile can vary by $\sim 9-16$ per cent between the observed wavelengths of the $[\mathrm{O} \mathrm{II}]$ and $[\mathrm{O} \mathrm{III}]$ lines. In our science analysis, this will be taken into account when relevant.

The slit width was chosen between 1.0 and 1.3 arcsec to reach a compromise between optimizing the observing time, obtaining an adequate spectral resolution and avoiding significant flux loses. The slit was often wider than the seeing disc as a consequence (see Table 2). This introduces additional uncertainties for spatially unresolved sources on the kinematic measurements. On one hand, if the objects are clumpy, the image of a spatially unresolved clump will be smaller than the slit width and the instrumental profile at that particular spatial position will be narrower than the profile measured using the sky or arc lines. This would lead to an underestimation 
Table 2. Summary of the VLT FORS2 observations. Columns: (1) source name; (2) source redshift; (3) date of the observation (run in 2011 April); (4) type of observation, where NB indicates narrow band imaging, IB indicates intermediate band imaging, BB indicates broad-band imaging, LSS indicates long slit spectroscopy, and HST indicates HST WFPC2 imaging; (5) filter, or grism and slit combination; (6) the position angle of the long slit, anticlockwise from north (north through east); (7) the exposure time on source of the observation; (8) the FWHM of the seeing disc as measured from stars in the broad-band images; (9) the average FWHM seeing conditions calculated over the exposure time as measured by the DIMM station; (10) the kiloparsec to arcsec conversion.

\begin{tabular}{|c|c|c|c|c|c|c|c|c|c|}
\hline $\begin{array}{l}\text { Name } \\
\text { (1) }\end{array}$ & $\begin{array}{c}z \\
(2)\end{array}$ & $\begin{array}{l}\text { Night } \\
\text { (3) }\end{array}$ & $\begin{array}{l}\text { Obs. } \\
(4)\end{array}$ & $\begin{array}{l}\text { Filter/grism } \\
\text { (5) }\end{array}$ & $\begin{array}{l}\mathrm{PA}\left({ }^{\circ}\right) \\
(6)\end{array}$ & $\begin{array}{l}\text { Exp. (s) } \\
\text { (7) }\end{array}$ & $\begin{array}{c}\text { Seeing }(\operatorname{arcsec})^{\mathrm{im}} \\
(8)\end{array}$ & $\begin{array}{c}\text { Seeing }(\operatorname{arcsec})^{\text {dimm }} \\
\text { (9) }\end{array}$ & $\begin{array}{c}\mathrm{kpc} \operatorname{arcsec}^{-1} \\
(10)\end{array}$ \\
\hline SDSS J0903+02 & 0.329 & $\begin{array}{l}28 \\
28 \\
28 \\
28 \\
28\end{array}$ & $\begin{array}{l}\text { BB } \\
\text { NB } \\
\text { LSS } \\
\text { LSS } \\
\text { LSS } \\
\text { HST }\end{array}$ & $\begin{array}{l}\text { v_HIGH } \\
\text { H_Alpha/4500+61 } \\
\text { 600RI } 1.3 \operatorname{arcsec} \text { slit } \\
\text { 600RI } 1.3 \operatorname{arcsec} \text { slit } \\
\text { 300I } 1.0 \text { arcsec slit } \\
\text { F814W }\end{array}$ & $\begin{array}{r}-65.5 \text { (PA1) } \\
63.4 \text { (PA2) } \\
-65.5 \text { (PA1) }\end{array}$ & $\begin{array}{r}730 \\
900 \\
4200 \\
1400 \\
2800 \\
1200\end{array}$ & $\begin{array}{l}0.81 \pm 0.03 \\
0.95 \pm 0.02\end{array}$ & $\begin{array}{l}0.88 \pm 0.05 \\
0.89 \pm 0.05 \\
1.00 \pm 0.29 \\
1.05 \pm 0.22 \\
0.93 \pm 0.24\end{array}$ & 4.71 \\
\hline SDSS J0950+01 & 0.404 & $\begin{array}{l}27 \\
27 \\
27\end{array}$ & $\begin{array}{l}\text { BB } \\
\text { IB } \\
\text { LSS }\end{array}$ & $\begin{array}{l}\text { v_HIGH } \\
\text { FILT_530_25 } \\
\text { 600RI 1.3 } \operatorname{arcsec} \text { slit }\end{array}$ & 9.9 & $\begin{array}{r}600 \\
900 \\
4200\end{array}$ & $\begin{array}{l}0.96 \pm 0.05 \\
1.05 \pm 0.04\end{array}$ & $\begin{array}{l}1.09 \pm 0.12 \\
1.16 \pm 0.11 \\
1.05 \pm 0.13\end{array}$ & 5.38 \\
\hline SDSS J1017+03 & 0.453 & $\begin{array}{l}28 \\
28\end{array}$ & $\begin{array}{l}\text { BB } \\
\text { LSS }\end{array}$ & $\begin{array}{l}\text { v_HIGH } \\
\text { 300I } 1.0 \operatorname{arcsec} \text { slit }\end{array}$ & 37.7 & $\begin{array}{r}600 \\
2100\end{array}$ & $0.74 \pm 0.03$ & $\begin{array}{l}0.69 \pm 0.02 \\
0.71 \pm 0.06\end{array}$ & 5.76 \\
\hline SDSS J1247+01 & 0.427 & $\begin{array}{l}25 \\
25 \\
25\end{array}$ & $\begin{array}{l}\text { BB } \\
\text { NB } \\
\text { LSS }\end{array}$ & $\begin{array}{l}\text { v_HIGH } \\
\text { He I+53 } \\
600 \text { RI } 1.3 \operatorname{arcsec} \text { slit }\end{array}$ & 60.7 & $\begin{array}{r}600 \\
900 \\
1443\end{array}$ & $\begin{array}{l}1.20 \pm 0.02 \\
1.24 \pm 0.05\end{array}$ & $\begin{array}{l}1.35 \pm 0.08 \\
1.36 \pm 0.12 \\
1.50 \pm 0.21\end{array}$ & 5.56 \\
\hline SDSS J1336-00 & 0.416 & $\begin{array}{l}26 \\
26 \\
26\end{array}$ & $\begin{array}{l}\text { BB } \\
\text { IB } \\
\text { LSS }\end{array}$ & $\begin{array}{l}\text { v_HIGH } \\
\text { FILT_530_25 } \\
\text { 600RI 1.0 arcsec slit }\end{array}$ & 57.4 & $\begin{array}{r}600 \\
300 \\
2800\end{array}$ & $\begin{array}{l}0.66 \pm 0.05 \\
0.63 \pm 0.02\end{array}$ & $\begin{array}{l}0.69 \pm 0.07 \\
0.84 \pm 0.07 \\
0.62 \pm 0.07\end{array}$ & 5.47 \\
\hline SDSS J1416-02 & 0.305 & $\begin{array}{l}27 \\
27 \\
27\end{array}$ & $\begin{array}{l}\text { BB } \\
\text { IB } \\
\text { LS }\end{array}$ & $\begin{array}{l}\text { v_HIGH } \\
\text { FILT_485_37 } \\
\text { 600RI } 1.3 \operatorname{arcsec} \text { slit }\end{array}$ & 90.0 & $\begin{array}{r}600 \\
900 \\
2800\end{array}$ & $\begin{array}{l}1.10 \pm 0.05 \\
1.04 \pm 0.04\end{array}$ & $\begin{array}{l}1.01 \pm 0.07 \\
0.95 \pm 0.04 \\
0.84 \pm 0.08\end{array}$ & 4.47 \\
\hline
\end{tabular}

of the intrinsic FWHM of the emission lines, which is the result of subtracting the instrumental profile in quadrature. The resulting uncertainties have been carefully taken into account and will be mentioned when relevant.

\subsection{Auxiliary Hubble Space Telescope (HST) data}

SDSS J0903+02 and SDSS J0923+01 have also been observed using the Wide Field and Planetary Camera 2 on the HST. These data were retrieved from the Hubble Legacy Archive (HLA). The images were obtained for the HST programme with identification 10880 and principal investigator Henrique Schmitt. In both cases the broad-band F814W filter was used.

\section{ANALYSIS AND RESULTS}

Figs 1-10 show our FORS2 images along with the corresponding HST image where available. Sections of the two-dimensional spectra are also shown to highlight extended nebular features, if detected.

In addition, in Fig. 12 we show emission line spatial profiles (usually [O III] $\lambda$ 5007), with the spatial point spread function during each observation also plotted for comparison. The spatial profiles of the emission lines were derived by extracting a one-dimensional spectrum at each spatial position along the slit, and by then integrating across the entire velocity profile of the emission line, or by fitting one or more Gaussian profiles to the line velocity profile.

Table 3 summarizes the general results of our analysis that will be described in detail below.

\subsection{Notes on individual objects}

\subsubsection{SDSS J0903+02}

The VLT and HST broad-band images of this QSO2 show a tadpole morphology comprising a spatially asymmetric light profile, with several bright knots along $\mathrm{PA} \sim 110^{\circ}$ in the central $\sim 3 \operatorname{arcsec}$ (14 kpc; Fig. 1). A low surface brightness tail along this same PA extends $\sim 16 \operatorname{arcsec}(75 \mathrm{kpc}$ ) from the position of the quasar. Very faint, diffuse emission is also seen to extend $\sim 16 \operatorname{arcsec}(75 \mathrm{kpc})$ to the north-east. In addition, a faint tongue also extends $\sim 3$ arc$\sec (14 \mathrm{kpc})$ to the south-east. The narrow band image, containing [O III $] \lambda 5007$ and continuum emission, reveals a morphology that is broadly similar to that seen in the broad-band images.

A visual inspection of our spectra reveals spatially extended line emission along both slit position angles (see Fig. 1). This is also obvious in Fig. 12 where the [O III] spatial profile is shown in 

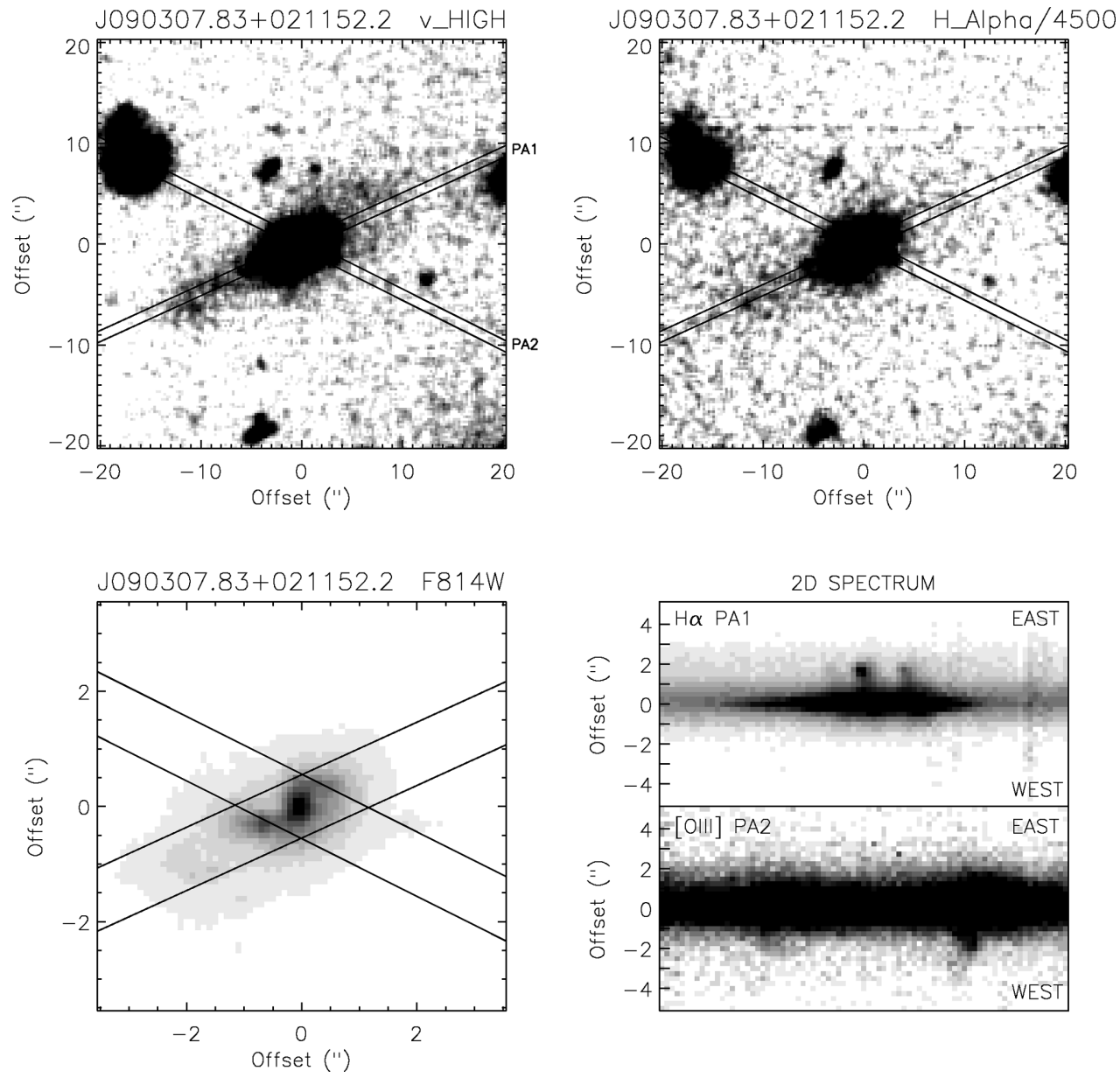

Figure 1. Images, the $[\mathrm{O}$ III $] \lambda \lambda 4959,5007$ line profile, and sections of the two-dimensional spectrum of SDSS J090307.83+021152.2. North is up and east is left in this and all remaining figures.

comparison with the seeing profile. Along slit PA1 $=-65.5$, the emission is dominated by a spatially unresolved central source. In addition, we detect a compact feature of line emitting gas in $\mathrm{H} \alpha$, [N $\mathrm{II}],[\mathrm{O}$ III], $\mathrm{H} \beta$ and [O II], located $1.8 \operatorname{arcsec}(8 \mathrm{kpc})$ South East from the quasar. Showing line ratios $\left[\mathrm{O}_{\mathrm{III}}\right] \lambda 5007 / \mathrm{H} \beta=1.5 \pm 0.2$ and $\left[\mathrm{N}_{\mathrm{II}}\right] \lambda 6583 / \mathrm{H} \alpha=0.47 \pm 0.05$, this feature falls within the 'composite' region defined by Kewley et al. (2001) in the Baldwin, Philips \& Televich (1981) (BPT) diagram. Strong underlying continuum is detected possibly associated with this knot (although it extends further out, so this is not certain). The lines are very narrow with FWHM $\lesssim 200 \mathrm{~km} \mathrm{~s}^{-1}$, taking into account possible slit effects (see Section 3.1.1). Based on the compact morphology (at least along the slit), the apparent spatial detachment from the nuclear region, the narrow lines and the composite line ratios, we argue this is most likely a star-forming object within the quasar ionization cone. The lack of spatial information perpendicular to the slit does not allow us to differentiate whether it could be a star-forming companion nucleus, a giant star-forming region or even a star-forming tidal feature, spatially unresolved along the slit.

The lines are extended on both sides of the QSO2 along slit PA2 $=63.4^{\circ}$ (Fig. 1). Very faint (not plotted in this figure due to its weakness) [O III] reaches a maximum extension of 4.8 arcsec $(22 \mathrm{kpc})$ from the quasar towards the West. The [O III] $\lambda 5007 / \mathrm{H} \beta$ ratio is $1.8 \pm 0.2$, indicating relatively low excitation, but insufficient by itself to discriminate between ionization by stars or the AGN.
Because it appears as an extended nebula connected with the QSO, we classify this as an extended emission line region (EELR). ${ }^{1}$ It shows narrow lines with $\mathrm{FWHM} \leq 180 \mathrm{~km} \mathrm{~s}^{-1}$.

\subsubsection{SDSS J0923+01}

The broad-band morphology of the host galaxy of this QSO2 was described in detail by Bessiere et al. (2012); based on deep Gemini Multi-Object Spectrograph broad-band optical images, they found a strong morphological disturbance. Our FORS2 optical image shows a galaxy morphology that is elongated along a PA $\sim-45^{\circ}$, with low surface brightness features that resemble tails and broad fans (Fig. 7). In addition, the central few arcsec shows a lopsided flux distribution.

Modelling of the stellar populations in the host galaxy of this QSO2 reveals a large contribution from a young stellar population with an age of between 50 and $100 \mathrm{Myr}$ (Bessiere et al. in preparation).

The spatial profile of the emission lines along the slit $\mathrm{PA}=40.9^{\circ}$ is dominated by a spatially unresolved central component (Fig. 12) with FWHM $\sim 0.61$ arcsec, consistent within the errors with the

\footnotetext{
${ }^{1}$ Throughout this paper, we define an EELR as a spatially extended nebula of line-emitting gas, which shows a clear, continuous physical connection to the line-emitting gas in the galaxy's nucleus.
} 

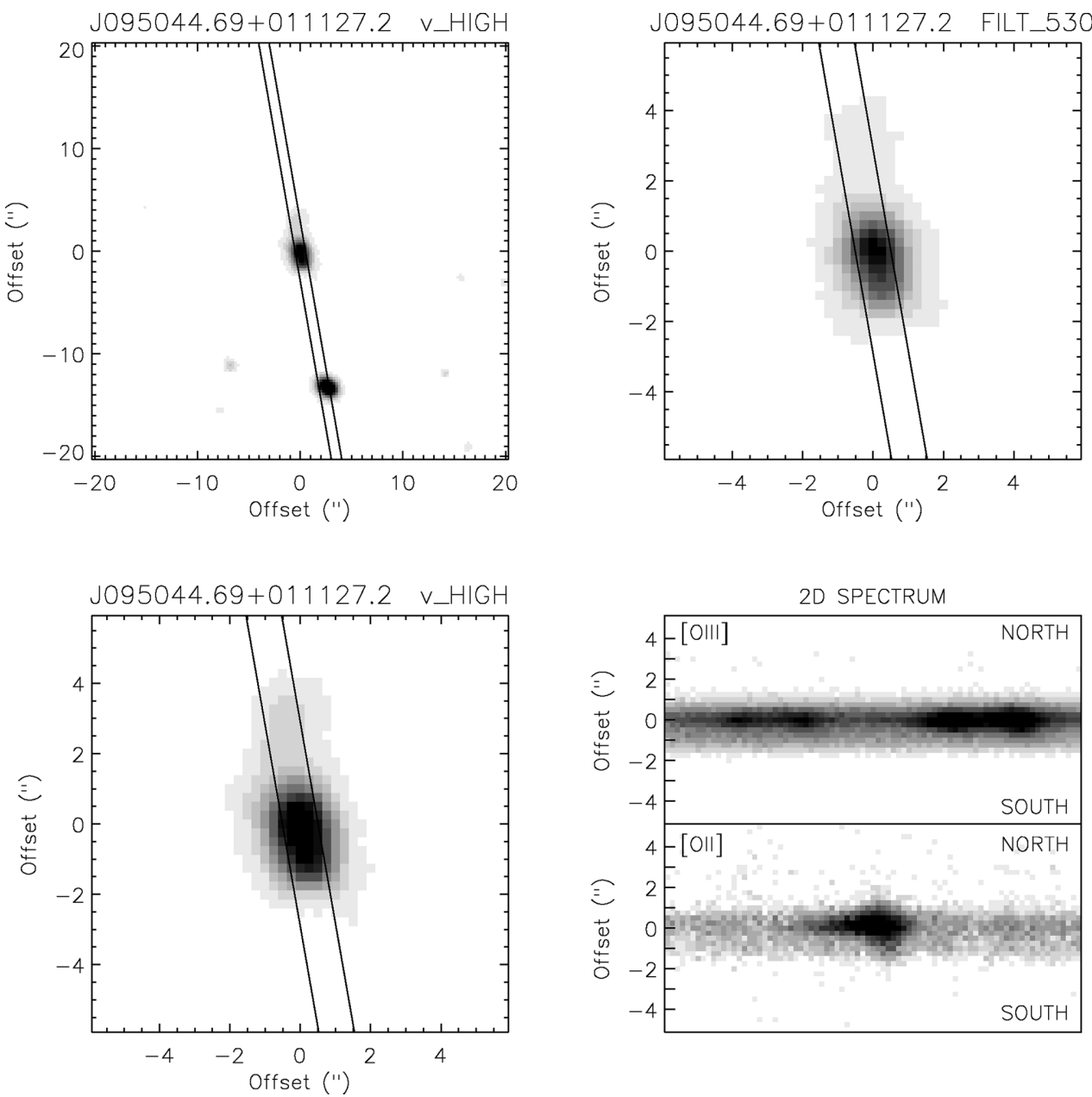

Figure 2. Images, the [O III] $\lambda \lambda 4959,5007$ line profile, and sections of the two-dimensional spectrum of SDSS J095044.69+011127.2

seeing values derived from broad-band image $(0.66 \pm 0.06$ arcsec; Table 2). In addition, both [O III] $\lambda 5007$ and $\mathrm{H} \alpha$ show a faint and compact (along the slit) feature of narrow emission lines (FWHM $<$ $200 \mathrm{~km} \mathrm{~s}^{-1}$, taking into account possible slit effects). It is located at $2 \operatorname{arcsec}(10 \mathrm{kpc})$ north-east of the AGN, blueshifted by $550 \mathrm{~km} \mathrm{~s}^{-1}$ (ignoring slit effects) relative to the nuclear emission. Continuum is not detected at the location of the knot. The object is clearly seen in the two-dimensional spectrum (Fig. 1) and also as a faint excess above the seeing profile in Fig. 12.

This knot shows [O $\mathrm{III}] \lambda 5007 / \mathrm{H} \beta \gtrsim 6$ and $\left[\mathrm{N}_{\mathrm{II}}\right] / \mathrm{H} \alpha \lesssim 0.2$ ratios that are suggestive of stellar photoionization (e.g. Kewley et al. 2001). The compact appearance along the slit, the narrow lines and the line ratios suggest that this is a star-forming object.

Also within the slit is the unrelated irregular galaxy SDSS J092317.57+010136.6 (see Fig. 7) at $\sim 11$ arcsec to the south-west (SW). The detected [O III] $\lambda \lambda 4959,5007$ and $\mathrm{H} \alpha$ lines place it at $z=0.191$.

\subsubsection{SDSS J0950+01}

This HLSy2 shows a strongly asymmetric light profile in its broadband image (Fig. 2). A low surface brightness tail extends $\sim 4$ arcsec $(22 \mathrm{kpc})$ north from the quasar, along a similar position angle to that of the high surface brightness regions of the galaxy, and a faint tail (or possibly a poorly resolved companion nucleus) also extends $\sim 5 \operatorname{arcsec}(27 \mathrm{kpc})$ southward. The narrow band image, which samples [О II] $\lambda 3727$ and continuum emission, shows similar morphological structure.

$[\mathrm{O}$ II] is the only line confirmed to be spatially extended, while the [O III] profile is consistent with a seeing disc (Fig. 12) of FWHM $\sim 1.0$ arcsec. Seeing broadening at decreasing $\lambda$ (expected to be $\leq 16$ per cent, see Section 3.1.1) cannot explain this difference in the spatial profiles of $[\mathrm{O} \mathrm{II}]$ and $[\mathrm{O} \mathrm{III}]$. The $\left[\mathrm{O}_{\mathrm{II}}\right]$ spatial profile suggests that the line is emitted by an EELR associated with the HLSy2. The total observed extent is $4.5 \mathrm{arcsec}(24 \mathrm{kpc})$ and maximum extent from the continuum centroid of 2.3 arcsec $(12 \mathrm{kpc}$; Table 3$)$. The line is very narrow in the extended gas, with a total doublet FWHM $\lesssim 235 \mathrm{~km} \mathrm{~s}^{-1}$ and $215 \pm 45 \mathrm{~km} \mathrm{~s}^{-1}$ respectively at both sides of the continuum centroid, taking possible slit effects into account.

\subsubsection{SDSS J1014+02}

In the broad-band image, the host galaxy of this HLSy2 shows a cometary, asymmetrical light profile in its higher surface brightness region, with an arc (or tail) of low surface brightness emission extending north-east and then north-west, terminating in a knot of emission $\sim 3.25 \operatorname{arcsec}(21 \mathrm{kpc}$ ) north of the galaxy (Fig. 3).

Our spectroscopic data show that the [O III] spatial profiles along both position angles are dominated by the central spatially unresolved source. Along PA1 $=-5.9$, this appears somewhat narrower 

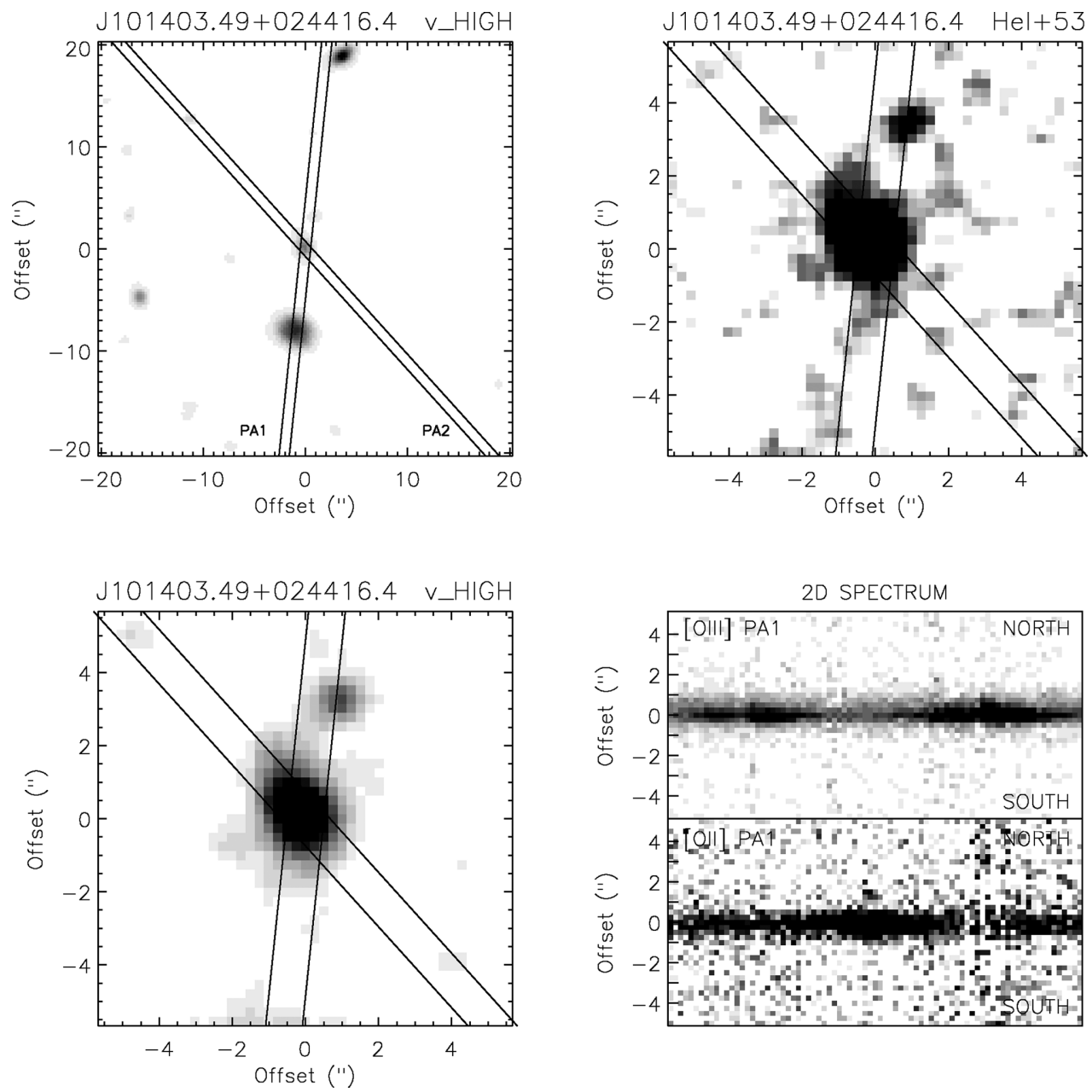

Figure 3. Images, the $[\mathrm{O}$ III] $\lambda \lambda 4959,5007$ line profile, and sections of the two-dimensional spectrum of SDSS J101403.49+024416.4.

than the seeing derived from the narrow band image (FWHM $\sim 0.64$ \pm 0.03 arcsec; Fig. 12). The slightly broader central [O II] spatial profile along PA1 is consistent with seeing broadening at shorter $\lambda$. Along PA2, [O II] appears slightly extended.

In addition, very low surface brightness (too faint to be plotted in Fig. 12), diffuse [O II] is detected along PA2 towards the SW up to $\sim 2.8$ arcsec or $18 \mathrm{kpc}$ from the continuum centroid. The lines are very narrow in this EELR. The doublet has FWHM $\lesssim 140 \mathrm{~km} \mathrm{~s}^{-1}$ in the EELR - in the most extreme case of maximum possible slit effects, the lines could have at most FWHM $=211 \pm 37 \mathrm{~km} \mathrm{~s}^{-1}$.

The galaxy 43 arcsec north-east from the AGN along PA2 detected in extended [O II] emission with $z=0.571$, confirming its association with the quasar. The galaxies 8 arcsec south and 19 arcsec north along PA1 unrelated star-forming galaxies at substantially different redshifts.

\subsubsection{SDSS J1017+03}

The broad-band image of this HLSy2 shows an extended low surface brightness amorphous halo and a compact knot located 3 arcsec $(17 \mathrm{kpc}) \mathrm{SW}$ of the nucleus (Fig. 4).

The $[\mathrm{O}$ III] $\lambda 5007$ spatial profile is dominated by a central unresolved source (Fig. 12). A faint excess above the seeing profile is detected at $\sim 1.2$ arcsec from the spatial centroid towards the NE. In addition, faint spatially extended emission is detected towards the
SW (too faint to be discerned in Fig. 12). It reaches a distance of $3.5 \operatorname{arcsec}(20 \mathrm{kpc})$ from the AGN (Fig. 4) where it seems to connect with the position of the knot seen in the broad-band image. This knot also shows line emission. The [O III] line is very narrow with FWHM $\lesssim 140 \mathrm{~km} \mathrm{~s}^{-1}$ (309 \pm 30 accounting for the most extreme possible slit effects) along its full extension and it is redshifted by up to $\sim 530 \mathrm{~km} \mathrm{~s}^{-1}$ relative to the [O III] emission at the position of peak flux. For the $[\mathrm{O}$ III $] \lambda 5007 / \mathrm{H} \beta$ ratio we obtain a $3 \sigma$ lower limit of $\geq 4$.

\subsubsection{SDSS J1247+01}

The broad-band image shows several other galaxies within 10 arcsec $(56 \mathrm{kpc})$ of the HLSy2, including a pair of galaxies located 4 arcsec (22 kpc) SW of the HLSy2 (Fig. 6). One member of this galaxy pair fell within the slit, and shows weak [O II] emission and a continuum break consistent with the $4000 \AA$ break at $z=0.42$, similar to that of the AGN host.

We detect a faint tidal tail extending $\sim 2$ arcsec west from the western-most of the companion galaxy pair. In addition, after processing the broad-band image using the "smoothed galaxy subtraction' technique of Ramos Almeida et al. (2011), we detect a tidal tail (or bridge) connecting the HLSy2 to its nearest companion galaxy (see lower panel in Fig. 6). We conclude that this is a triple merger/interacting system. 

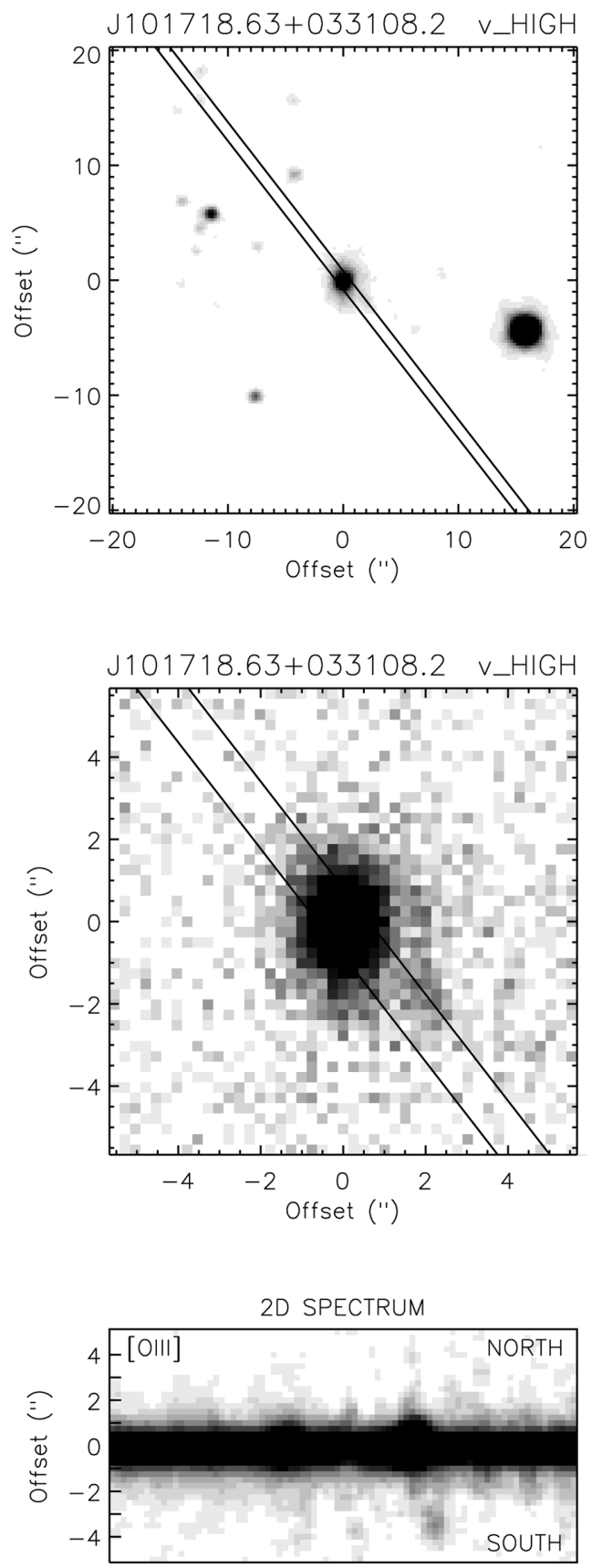

Figure 4. Images, the $[\mathrm{O} \mathrm{III}] \lambda \lambda 4959,5007$ line profile, and a section of the two-dimensional spectrum of SDSS J101718.63+033108.2

Our spectroscopic data show that the [O $\mathrm{II}$ ] and [O III] spatial profiles along $\mathrm{PA}=60.7$ are both spatially extended. The $[\mathrm{O}$ II] and [O III $]$ profiles are shown in Fig. 12 together with a seeing profile of FWHM $\sim 1.2$ arcsec. Both [O II] and [O III] show a clear excess above the seeing disc at both sides of the spatial centroid. This is also clear in the two-dimensional spectrum (Fig. 6). A visual inspection shows that extended faint $[\mathrm{O} \mathrm{II}]$ is detected along the slit up to $\sim 3$ arcsec NE from the continuum centroid $(\sim 17 \mathrm{kpc})$, with well differentiated kinematics. The lines appear spectrally unresolved with FWHM $\lesssim 120 \mathrm{~km} \mathrm{~s}^{-1}$ (slit effects do not affect this object). For [O III], the excess is clear towards the east.

This galaxy was detected by the FIRST survey $(20 \mathrm{~cm} / 1.4 \mathrm{GHz})$ with a flux density of $7.1 \pm 0.1 \mathrm{mJy}$. In addition to a bright radio core,

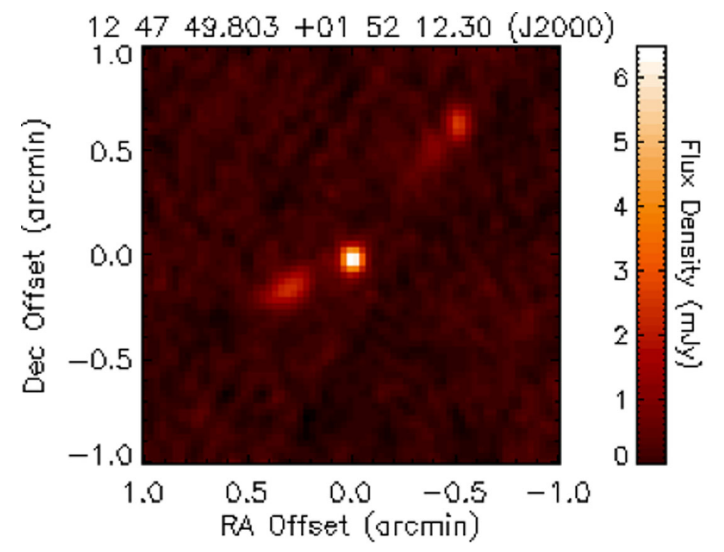

Figure 5. FIRST $20 \mathrm{~cm}(1.4 \mathrm{GHz})$ postage-stamp image of the radio-loud QSO2 SDSS J124749.79+015212.6. See also Lal \& Ho (2010).

the FIRST image also shows two diametrically opposed radio lobes with a separation of $\sim 70 \operatorname{arcsec}(400 \mathrm{kpc}$; Fig. 5). The substantially higher NVSS flux density of $34.1 \pm 1.6 \mathrm{mJy}(20 \mathrm{~cm} / 1.4 \mathrm{GHz})$ further attests to the presence of large-scale, low surface brightness radio emission (see also Lal \& Ho 2010).

The galaxy at $\sim 9$ arcsec to the north-east from the AGN has photometric $z=0.456 \pm 0.026$, according to the SDSS information. This suggests that it could also be associated with the quasar, but spectroscopic $z$ confirmation is needed.

\subsubsection{SDSS J1336-00}

This apparently isolated galaxy shows an elliptical compact morphology in our broad and intermediate band images (Fig. 8). No spatial structure is apparent, and there are no other bright galaxies visible within a radius of at least $80 \mathrm{kpc}$. In spite of this unremarkable appearance, the optical spectrum shows strong Balmer absorption lines and a strong Balmer break (Fig. 11), suggesting a burst of star formation occurred $\gtrsim 100 \mathrm{Myr}$ ago.

Although several strong nebular emission lines are detected in our spectrum, we find no evidence for any spatial extension along our slit PA. [O II] is not plotted for this object because the strong adjacent stellar features prevent an accurate measurement of the line flux at different spatial locations (Fig. 11), but there is no evidence of spatial extension for this line either. Indeed, the [O III] emission is rather more centrally peaked than the 5000-6000 $\AA$ continuum emission. It is also narrower (Fig. 12) than the seeing size implied by the images and DIMM values ( 0.6-0.7 arcsec; Table 2$)$.

\subsubsection{SDSS J1416-02}

The broad and narrow band images of this HLSy2 show no clear tidal features or possible interacting neighbours (Fig. 9). However, due to its non-uniform surface brightness distribution, with an elongation towards the north-west, we consider it plausible that this is a postinteracting system.

The long slit spectrum shows spatially extended emission lines emitted by an EELR (Figs 9 and 12), with the strongest line [O III] $\lambda 5007$ having a total extent of 6 arcsec $(27 \mathrm{kpc})$ and maximum extension from the continuum centroid of $\sim 3.25$ arcsec or $14.5 \mathrm{kpc}$. The spatial peaks of the emission lines have a small but significant offset of $\sim 0.3 \operatorname{arcsec}(1.3 \mathrm{kpc})$ east of the continuum peak. Across the observed spatial extent of the nebulosity, the $\left[\mathrm{O}_{\text {IIII }}\right] \lambda 5007 / \mathrm{H} \beta$ flux ratio is within the range $5-10$. The detection of the He II $\lambda 4686$ 

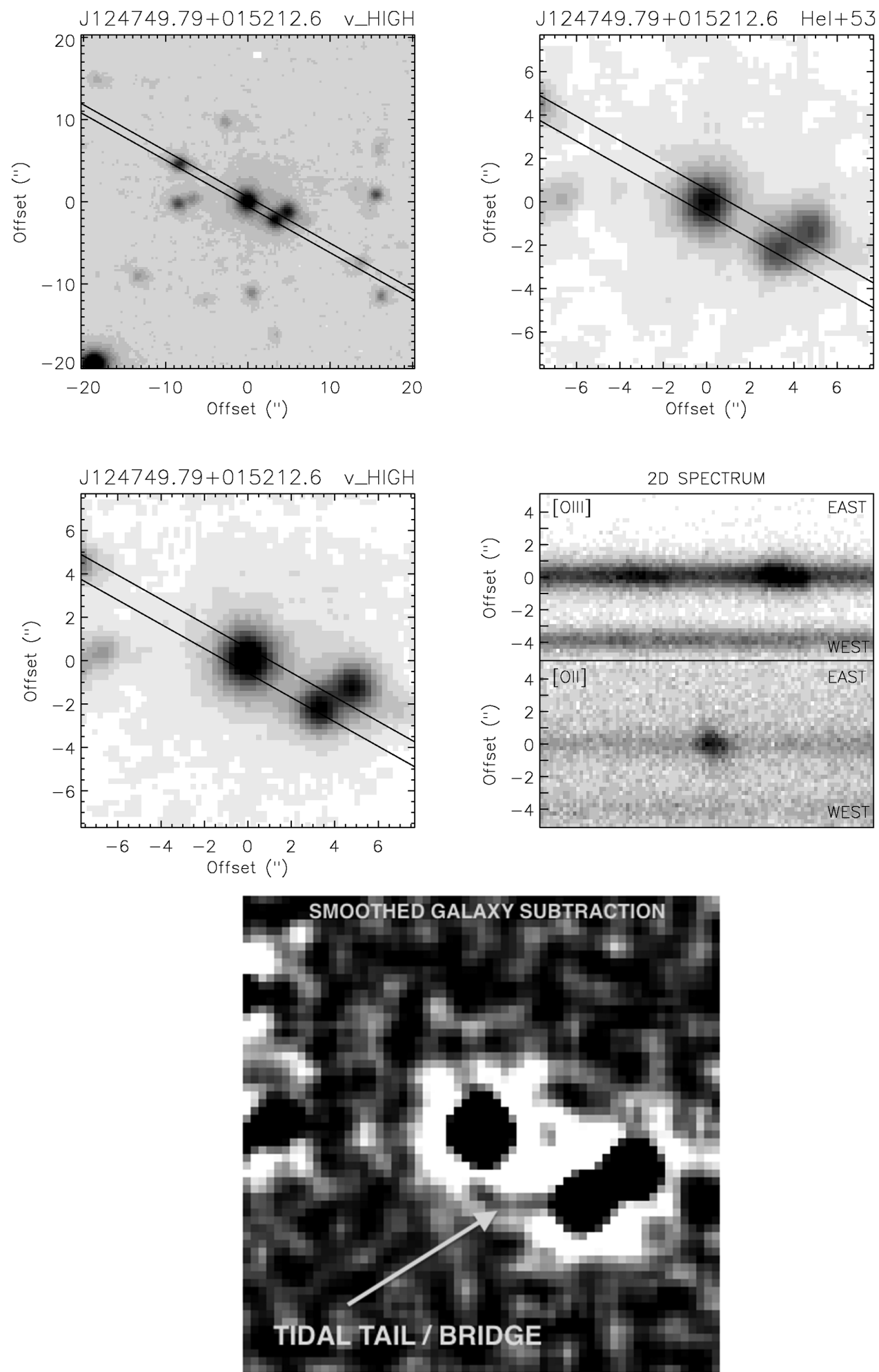

Figure 6. Images and the [O III] $\lambda \lambda 4959,5007$ line profile, of SDSS J124749.79+015212.6. The lower panel shows the central 15.25 arcsec $\times 15.25$ arcsec of broad-band image of the field around the HLSy2, after applying the 'smoothed galaxy subtraction' technique of Ramos Almeida et al. (2011). This analysis has revealed a tidal tail (or bridge) connecting the HLSy2 to its nearest companion galaxy, visible as a dark arc to the south and SW of the HLSy2. 

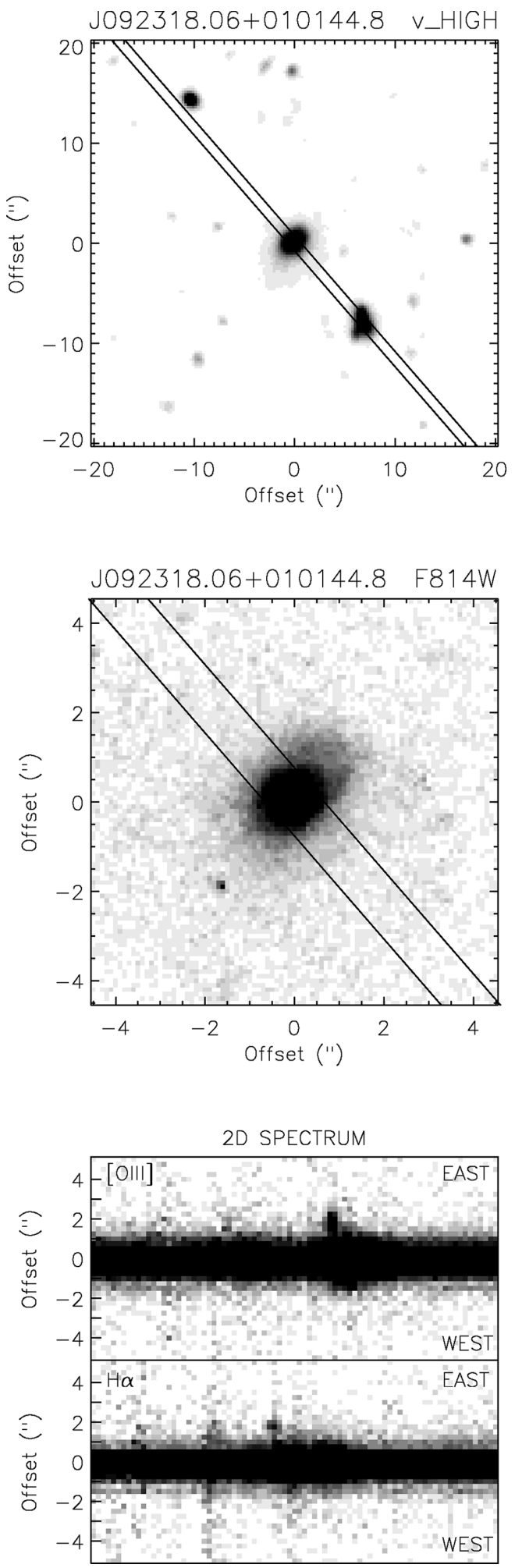

Figure 7. Images, the $\left[\mathrm{O}_{\mathrm{III}}\right] \lambda \lambda 4959,5007$ line profile, and sections of the two-dimensional spectrum of SDSS J092318.06+010144.8.

line on the Western side of the EELR confirms that the nebula is photoionized by the AGN.

The lines are very narrow in the EELR. [O III] is unresolved on the west side of the nebula, with FWHM $\lesssim 200 \mathrm{~km} \mathrm{~s}^{-1}$ ), while FWHM $\lesssim 250 \mathrm{~km} \mathrm{~s}^{-1}$ on the east side, taking slit effects into account.
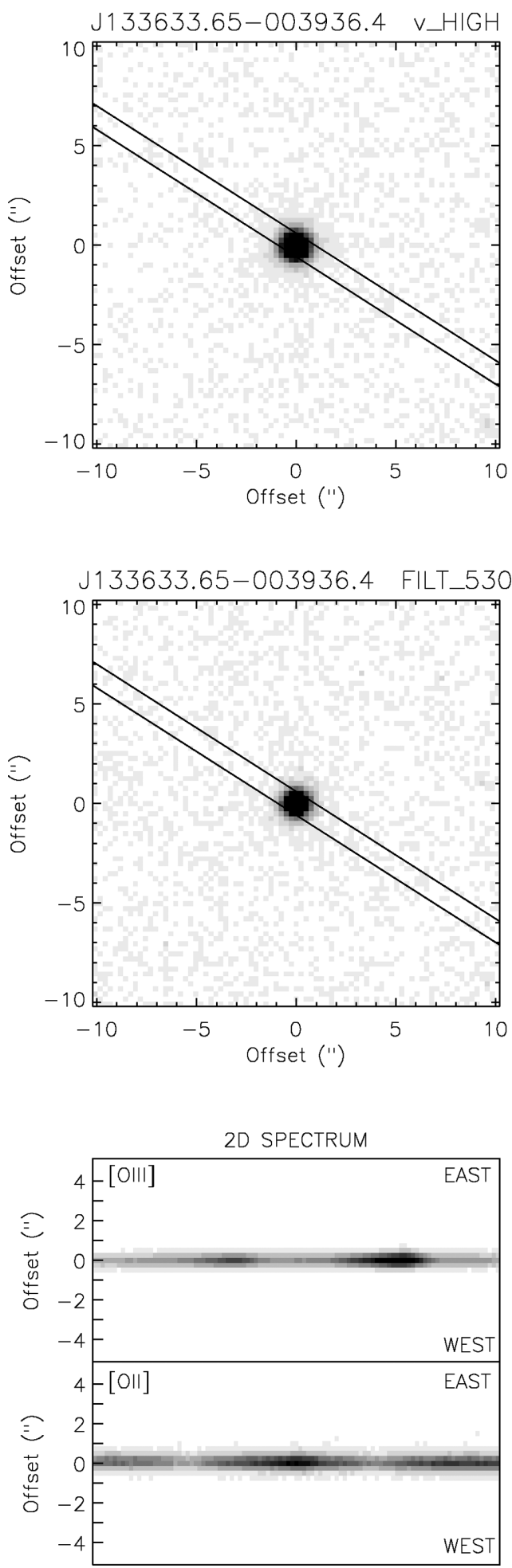

Figure 8. Images and the [O III] $\lambda \lambda 4959,5007$ line profile of SDSS J133633.65-003936.4.

\subsubsection{SDSS J1452+00}

The broad-band image of this HSy2 reveals an arm or tail of faint emission extending out to $\sim 4 \operatorname{arcsec}(18 \mathrm{kpc}$ ) north-west (Fig. 10). The association of this extended source is confirmed by its detection in $[\mathrm{O} \mathrm{III}]$ emission at a similar redshift to the HSy2. 

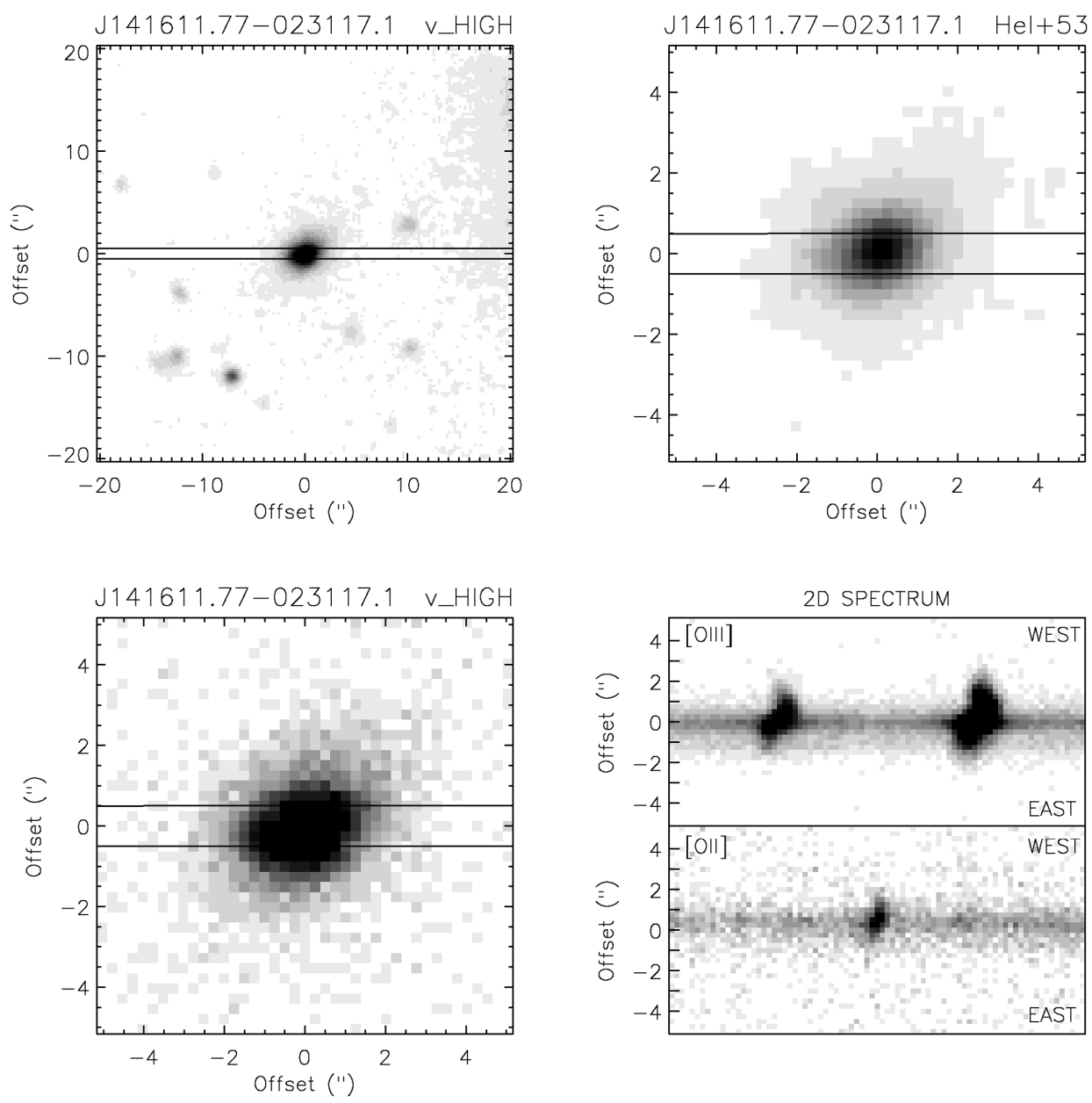

Figure 9. Images, the [O III] $\lambda \lambda 4959,5007$ line profile and sections of the two-dimensional spectrum of SDSS J141611.77-023117.1.

The [O III] spatial profile (Fig. 12) is dominated by a compact unresolved (FWHM $\sim 0.6$ arcsec) source. In addition, very faint [O III] extended emission (undetected in the figure) is detected up to $\sim 3.5$ arcsec or $16 \mathrm{kpc}$ from the continuum centroid (see twodimensional spectrum in Fig. 10). This emission overlaps with the tidal feature discussed above. The detection of relatively strong continuum suggests that it is forming stars. Unfortunately, the spectrum of this region is noisy and the line FWHM and ratios cannot be constrained in a useful way.

\section{OVERALL RESULTS AND DISCUSSION}

We have presented optical imaging and long slit spectroscopic observations of nine luminous type 2 AGN within the redshift range $0.3<z<0.6$. Most (6/9) are HSy2 and three are QSO2. This work expands a similar study by VM11 a towards somewhat less luminous AGN. Thus far we have described the results on an object-by-object basis. Here, we discuss the overall results of this study.

\subsection{Morphological signatures of mergers and interactions}

The host galaxies of our sample show a variety of optical morphologies, ranging from heavily disturbed morphologies suggestive of a recent or ongoing major merger, to morphologically regular and unremarkable systems.
Seven out of nine objects ( 78 per cent) show strong morphological evidence for interactions or mergers in the form of disturbed morphologies and/or peculiar features such as tidal features, amorphous haloes, compact emission line knots (see Table 3). The two remaining objects - SDSS J1336-00 (a QSO2) and SDSS J1416-02 (a HLSy2) - appear as isolated galaxies and have no unequivocal signs of mergers/interactions at the depth of our images. However, SDSS J1416-02 is associated with extended low surface brightness continuum emission, with a lopsided flux distribution that could be interpreted as a post-merger system (among other possible interpretations).

In comparison, the detection rate of interactions/mergers found by VM11a was $5 / 13$ objects (38 per cent). However, their fraction is a conservative lower limit (stated by the authors), because they had only shallow continuum images, and no narrow band emission line images.

Indeed, our detection rate of interaction signatures is equal to that found by Bessiere et al. (2012) in a complete sample of 20 SDSS selected QSO2 at $3<z<0.41$ (see also Villar-Martín et al. 2012) and also similar to that found for a control sample of quiescent (i.e. non active) early type galaxies of similar mass. Bessiere et al. (2012) found a significant difference in surface brightness for the interaction features (2 magnitudes brighter for the QSO2). They propose that the mergers witnessed in the comparison sample galaxies could have different progenitors, or that the interactions might be viewed at different stages. 

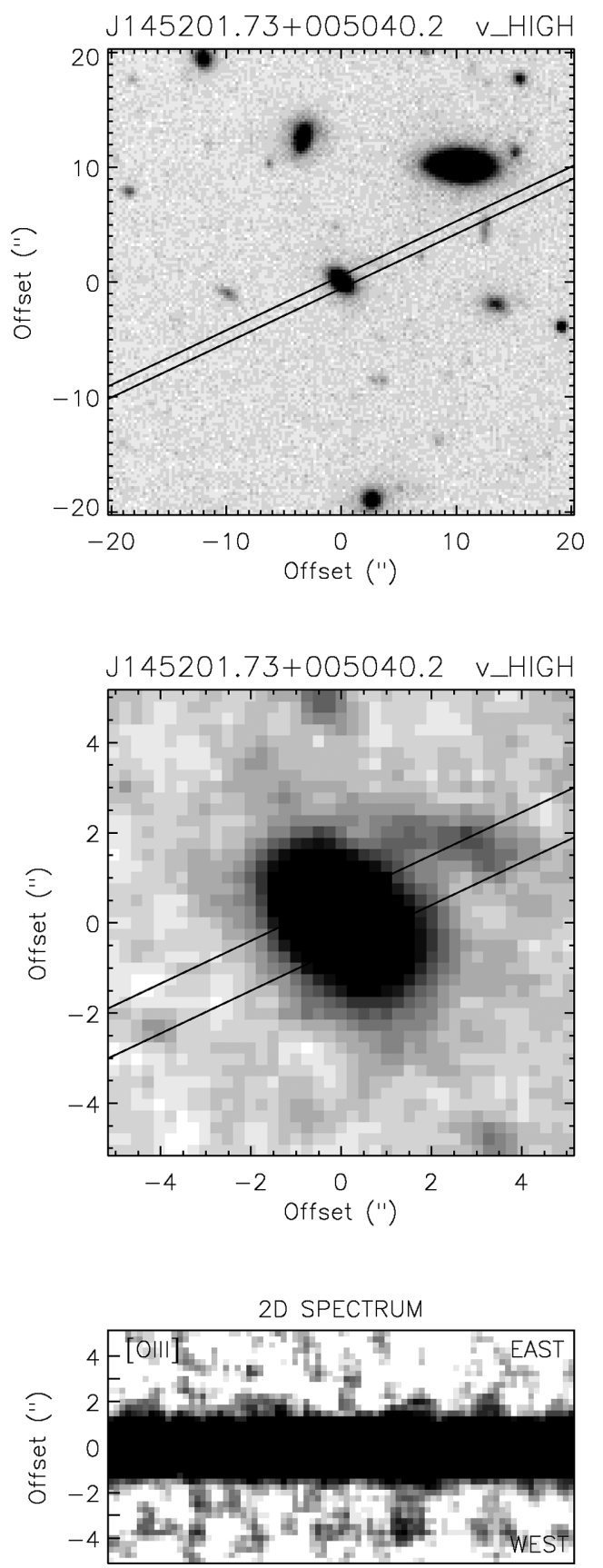

Figure 10. Images, the [O III] $\lambda \lambda 4959,5007$ line profile and a section of the two-dimensional spectrum of SDSS J145201.73+005040.2.

\subsection{Extranuclear line emission}

Our analysis shows that the spatial distribution of the emission lines is in general dominated by a compact spatially unresolved (i.e. consistent with the seeing disc) central source which emits very strong emission lines associated with the narrow line region. In addition, extranuclear line emission of much lower surface brightness is often detected due to structures of diverse nature: tidal tails, star-forming nuclei/knots/companions and extended ionized nebulae. The main results are summarized in Table 3. Specifically, we have detected extranuclear line emission for eight out of nine of our sample. The only exception is SDSS J1336+00, which, on the other hand, is an isolated galaxy with no obvious signs of mergers/interactions. The non-detection of extranuclear line emission might be due to a real absence of such emission. However, unlike for other objects, given the absence of peculiar/interesting features in the optical images the slit PA was chosen blindly so that extended lines along other PA might have been missed.

Our previous studies (VM11a; Villar-Martín et al. 2012) revealed extended emission lines associated with 7/15 QSO2 at similar $z$ although this is a gross lower limit, given that the slit was placed blindly for several objects with no obvious peculiar morphological features. On the other hand, we have shown here that $\left[\mathrm{O}_{\mathrm{II}}\right] \lambda 3727$ is often more extended (e.g. SDSS J0950+01, SDSS J1014+02, SDSS J1247+01) than [O III]. Our previous results were based on the analysis of the [O III] line only. The relatively higher [O II]/[O III] in the extended gas suggests that the extranuclear ionized gas has lower ionization level than the nuclear ionized gas. Moreover, while the gas in the central, often spatially unresolved region is preferentially photoionized by the active nucleus, the extranuclear gas is often at least partially photoionized by stars (see also McElroy et al. 2015). Both facts favour an easier detection of extranuclear line emission using low ionization lines such as [O II] compared with [O III].

Integral field spectroscopic works show that extended line emission is common in QSO2 at this $z$, although these authors studied objects with very high [O III] luminosities (Liu et al. 2013a).

Extranuclear emission line features other than EELR (companion nuclei, knots, tidal tails) are often identified (4/9 in our current sample or $10 / 24$ in the total sample including our prior studies). Thus, in $\sim 50$ per cent of the objects emission line structures whose nature is linked with mergers/interactions are detected.

EELR (which we differentiate from knots, tails, companion nuclei) are detected for $6 / 9$ objects. The maximum extension from the AGN is in the range $12-22 \mathrm{kpc}$, with mean (and median) values of $\sim 17 \mathrm{kpc}$. Liu et al. (2013a) detected extranuclear ionized nebulae via [O III] $\lambda 5007 \AA$ emission in most of the QSO2 they studied in a sample of 11 radio quiet QSO2 at similar $z$ to our sample. They measured radii of the ionized gas nebulae ranging from $r=7.5$ to $r=20 \mathrm{kpc}$, as seen from the $5 \sigma$ detection values. Their mean value is $r=14 \mathrm{kpc}$.

Thus, as shown by our previous studies, luminous type 2 AGN are associated with extranuclear emission line regions, which are often a complex mixture of tidal/interaction/companion features and EELR, with a mixture of excitation mechanisms (AGN-related processes and stellar photoionization) whose relative contribution varies spatially (see also McElroy et al. 2015). These regions therefore cannot be interpreted as a single gaseous structure where the morphology, kinematics and excitation are determined by a single mechanism.

\subsection{Ongoing star formation}

VM11a found evidence for recent star formation in the neighbourhood of 5/14 objects. All show signs of mergers/interactions. The star formation is happening in general in companion galaxies, knots, and/or nuclei. Definite (i.e. confirmed by the emission line spectrum) evidence for recent star formation is confirmed in two objects of the sample investigated here: SDSS 0903+02 and SDSS J0923+01, both with associated compact star-forming knots, and possibly also SDSS $1017+03$. All three also show evidence of interactions. These fractions are lower limits, given the obviously limited spatial coverage and the lack of sufficient line ratio information for diagnostics of the ionizing mechanisms. It is clear that at least part of the extended SF occurring in luminous type 2 AGN is triggered by mergers/interactions. A more complete study in two spatial 
Table 3. Summary of results. We specify for each object whether an EELR has been detected (column 2), and where the observational data allow, its nature (column 3). In columns 4 and 5 we give the maximum radial extension of the EELR from the AGN, in arcsec and kpc, respectively; in the case of physically distinct emission line sources (e.g. star-forming knots), we instead give their offset from the AGN. We also specify whether morphological signatures of galaxy interactions have been detected in our images (column 6).

\begin{tabular}{|c|c|c|c|c|c|}
\hline Galaxy & $\begin{array}{l}\text { Feature } \\
\text { (2) }\end{array}$ & $\begin{array}{l}\text { Spectrum } \\
\text { (3) }\end{array}$ & $\begin{array}{l}\text { Max. Ext. or Dist. } \\
(\operatorname{arcsec}) \\
(4)\end{array}$ & $\begin{array}{l}\text { Max. Ext. or Dist. } \\
(\mathrm{kpc}) \\
(5)\end{array}$ & $\begin{array}{c}\text { Interactions } \\
\text { (6) }\end{array}$ \\
\hline SDSS J0903+02 PA2+63.4 & EELR & $?$ & 4.8 & 22 & Yes \\
\hline PA1 -65.5 & SF compact object & Composite & 1.8 & 8 & \\
\hline SDSS J0923+01 PA-40.9 & SF compact object & SF & 2 & 10 & Yes \\
\hline SDSS J0950+01 PA-9.9 & EELR & $?$ & 2.3 & 12 & Yes \\
\hline SDSS J1014+02 PA-42.1 & EELR & $?$ & 2.8 & 18 & Yes \\
\hline SDSS J1017+03 PA-37.7 & EELR & $?$ & 3.0 & 17 & Yes \\
\hline PA-37.7 & Compact object & $?$ & 3.5 & 20 & \\
\hline SDSS J1247+01 PA-60.7 & EELR & $?$ & 3 & 17 & Yes \\
\hline SDSS J1336+00 PA-57.4 & No & - & - & - & No \\
\hline SDSS J1416-02 PA90 & EELR & AGN & 3.25 & 14.5 & Maybe \\
\hline SDSS J1452+00 PA64.6 & Tidal tail? & $?$ & 3.5 & 16 & Yes \\
\hline
\end{tabular}

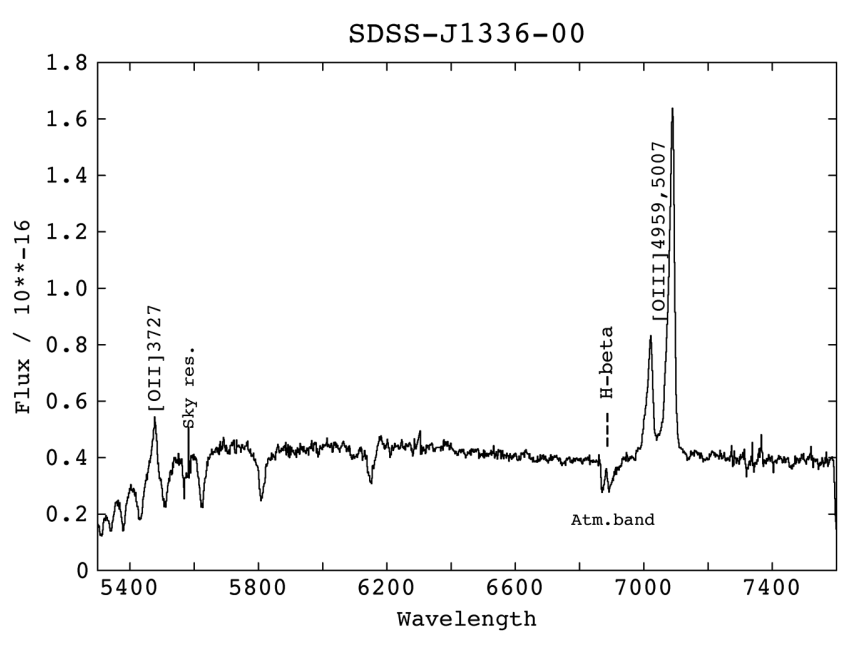

Figure 11. Nuclear spectrum of SDSS J1336-00. Strong Balmer absorption lines and the Balmer break suggest the presence of a post-starburst stellar population of $\gtrsim 100 \mathrm{Myr}$ of age. $\mathrm{H} \beta$ is affected by an atmospheric band. The flux is in units of $\times 10^{-16} \mathrm{erg} \mathrm{s}^{-1} \mathrm{~cm}^{-2} \operatorname{arcsec}^{-2}$.

dimensions would probably reveal recent star formation at least in some objects (e.g. disk galaxies) not necessarily related to this type of processes (e.g. McElroy et al. 2015). To ascertain the presence of a young stellar population in the nuclear regions it would be necessary to fit the optical nuclear spectrum with spectral synthesis modelling techniques (e.g. Tadhunter et al. 2011; Bessiere et al. in preparation).

\section{CONCLUSIONS}

We have presented optical imaging and long slit spectroscopic observations of nine luminous type $2 \mathrm{AGN}$ within the redshift range $0.3<z<0.6$ based on VLT FORS2 data. Six out of the nine objects are HSy2 $\left(\log \left(\frac{L_{[\mathrm{Oml}}}{\mathrm{L}_{\odot}}\right)<8.3\right)$ and three are QSO2 $\left(\log \left(\frac{L_{[\mathrm{Oml}}}{\mathrm{L}_{\odot}}\right)>8.3\right)$. This is an extension of the work presented in Villar-Martín et al. (2011a,b), who studied mostly QSO2, and we have thus enlarged the sample towards somewhat less luminous type 2 AGN.

(i) Signatures of mergers/interaction. Seven out of nine objects (78 per cent) show clear morphological evidence for interactions or mergers in the form of disturbed morphologies and/or peculiar features such as tidal tails, amorphous haloes, compact emission line knots, etc. This rate of interaction is consistent with other relevant studies of (more luminous) QSO2 at similar $z$, suggesting the merger rate is independent of the AGN luminosity at the high end of the AGN luminosity function (QSO2 and HLSy2).

(ii) Extended line emission. The emission line spatial profiles are dominated by a bright compact, usually spatially unresolved central source. In addition, much fainter, extranuclear emission line features are detected associated with $8 / 9$ objects. They are of a diverse nature: $\operatorname{EELR}$ (6/9 objects) of typical radial sizes $12-22 \mathrm{kpc}$ (consistent with related works focused on more luminous objects) as well as features related to mergers/interactions such as star-forming compact knots and tidal tails (4/9). There is a mixture of excitation mechanisms (AGN-related processes and stellar photoionization) whose relative contribution varies spatially. While the emission line spectrum of the ionized gas near the central engine $(R \lesssim$ few $\mathrm{kpc})$ is clearly excited by AGN-related processes, stellar photoionization can also be present in the extranuclear ionized gaseous structures. Moreover, we have found evidence, based on the $\left[\mathrm{O}_{\mathrm{II}}\right] /[\mathrm{O} \mathrm{III}]$ line flux ratio, that the extranuclear ionized gas is often in a lower ionization state than the nuclear ionized gas. In addition, the [O II] emission is often more spatially extended than [O III], suggesting that low ionization lines (such as $[\mathrm{O} \mathrm{II}]$ ) might be relatively more efficient than [O $\mathrm{III}]$ for detecting extranuclear and extended emission line structures in QSO2.

\section{ACKNOWLEDGEMENTS}

This study is based on observations carried out at the European Southern Observatory (Paranal, Chile) with FORS2 on VLT UT1 (programme 087.B-0034). We thank the staff at Paranal Observatory for their support during the observations. AH acknowledges Fundação para a Ciência e a Tecnologia (FCT) support through UID/FIS/04434/2013, and through project FCOMP-01-0124-FEDER-029170 (Reference FCT PTDC/FISAST/3214/2012) funded by FCT-MEC (PIDDAC) and FEDER (COMPETE), in addition to FP7 project PIRSES-GA-2013612701. AH also acknowledges a Marie Curie Fellowship cofunded by the FP7 and the FCT (DFRH/WIIA/57/2011) and FP7/FCT Complementary Support grant SFRH/BI/52155/2013. 

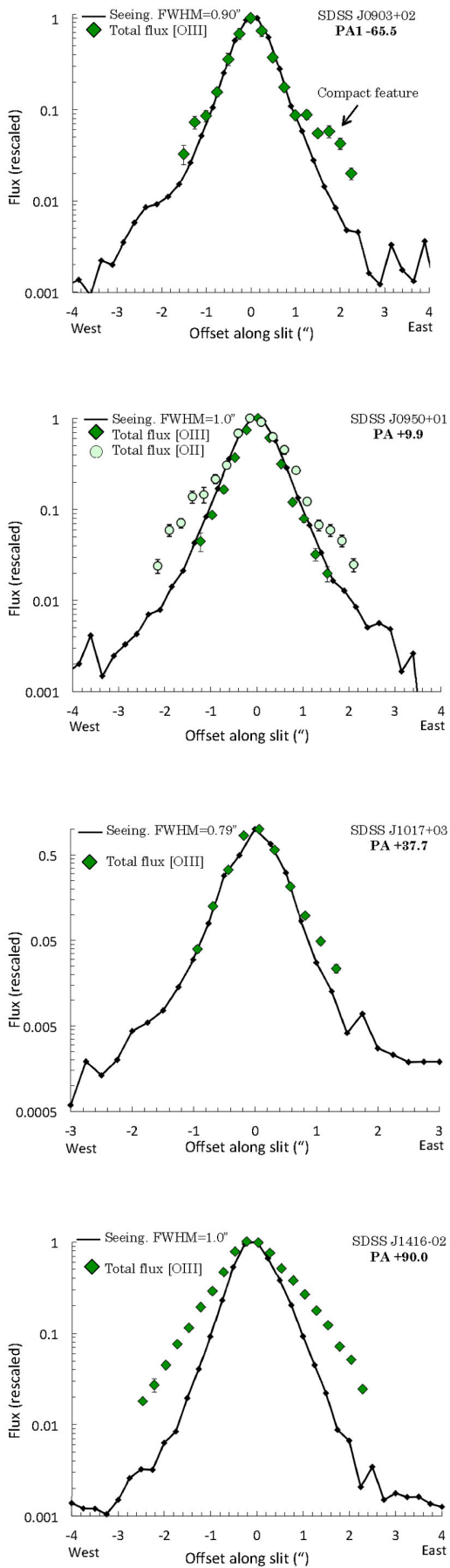
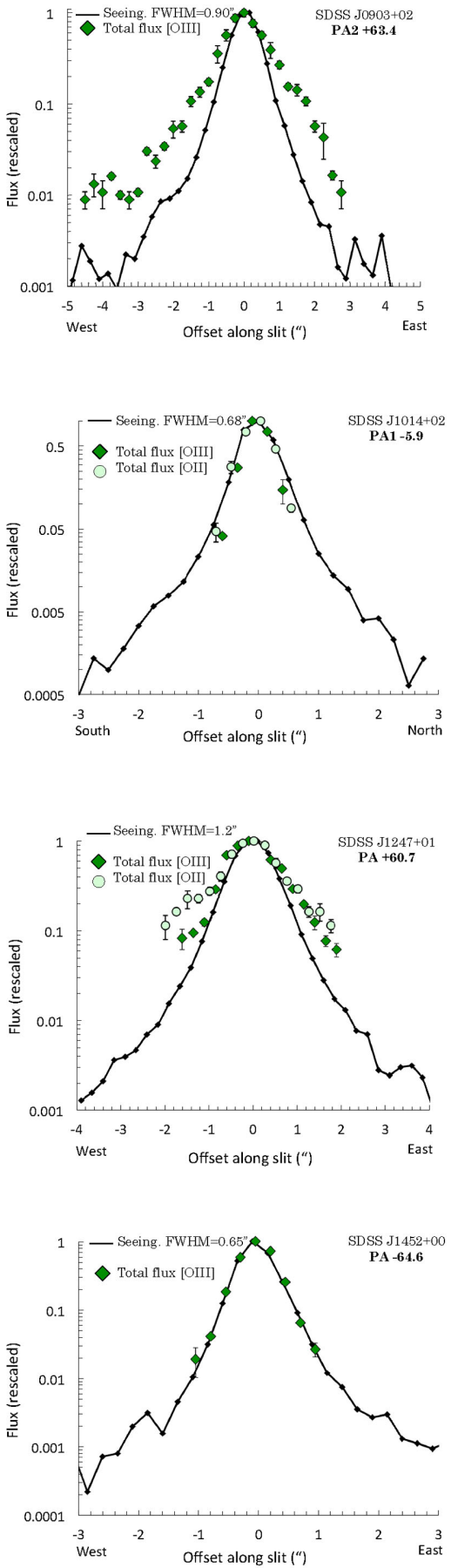
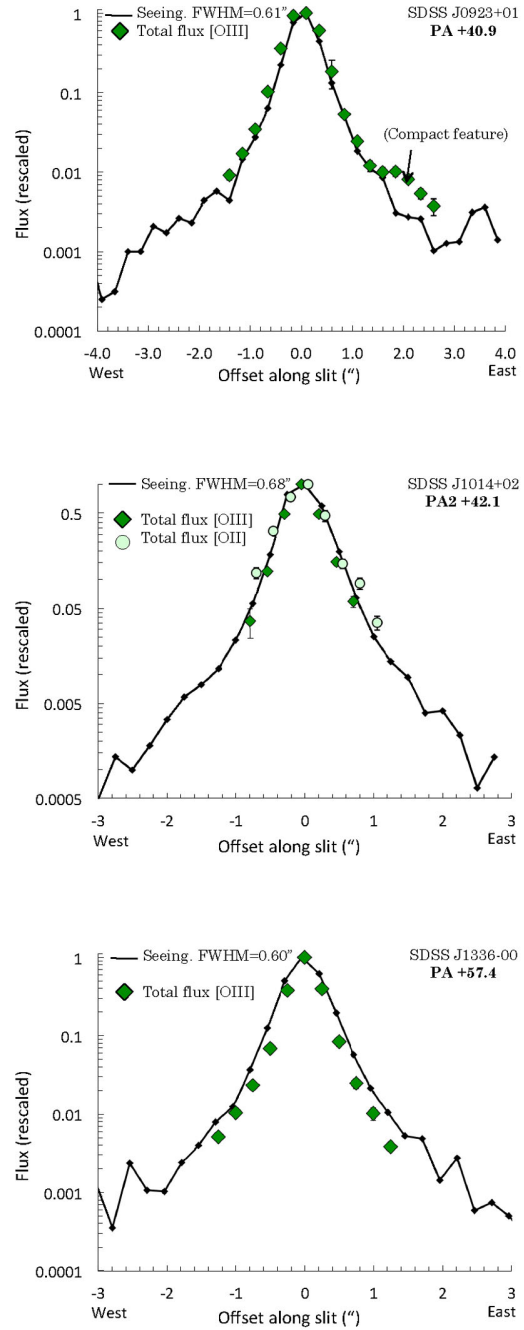

Figure 12. Spatial profile of the $\left[\mathrm{O}_{\mathrm{III}}\right] \lambda 5007$ emission (also [O II $] \lambda 3727$ in some cases) along the slit, compared against the spatial profile of the seeing disc.

MVM and SA acknowledge support from the Spanish Ministerio de Economía y Competitividad through the grants AYA2012-32295 and AYA-2012-39408-C02-01. CRA is supported by a Marie Curie Intra European Fellowship within the 7th European Community Framework Programme (PIEF-GA-2012-327934). RGD acknowledges support through the grant AYA2010-15081.

\section{REFERENCES}

Alexandroff R. et al., 2013, MNRAS, 435, 3306

Appenzeller I. et al., 1998, Messenger, 94, 1
Arribas S., Colina L., Bellocchi E., Maiolino R., Villar-Martín M., 2014, A\&A, 568, A14

Baldwin J., Philips M., Televich R., 1981, PASP, 93, 5

Becker R. H., White R. L., Helfand D. J., 1995, ApJ, 450, 559

Bessiere P. S., Tadhunter C. N., Ramos Almeida C., Villar-Martín M., 2012, MNRAS, 426, 276

Bessiere P. S., Tadhunter C. N., Ramos Almeida C., Villar-Martín M., 2014, MNRAS, 438, 1839

Bian W. H., 2007, in Ho L. C., Wang J.-M., eds, Astronomy Society of the Pacific Conference Series Vol. 373, The Central Engine of Active Galactic Nuclei. Astron. Soc. Pac., San Francisco, p. 675 
Condon J. J., Cotton W. D., Greisen E. W., Yin Q. F., Perley R. A., Taylor G. B., Broderick J. J., 1998, AJ, 115, 1693

Croft S. et al., 2006, ApJ, 647, 1040

Halpern J. P., Turner T. J., George I. M., 1999, MNRAS, 307, L47

Harrison C. M., Alexander D. M., Mullaney J. R., Swinbank A. M., 2014, MNRAS, 441, 3306

Heckman T. M., Smith E. P., Baum S. A., van Breugel W. J. M., Miley G. K., Illingworth G. D., Bothun G. D., Balick B., 1986, ApJ, 311, 526

Humphrey A., Villar-Martín M., Sánchez S. F., Martínez-Sansigre A., Delgado R. G., Pérez E., Tadhunter C., Pérez-Torres M. A., 2010, MNRAS, 408, L1

Kewley L., Dopita M., Sutherland R., Heisler C. Trevena J., 2001, AJ, 556, 121

Lal D. V., Ho L. C., 2010, AJ, 139, 1089

Liu G., Zakamska N. L., Greene J. E., Nesvadba N., Liu X., 2013a, MNRAS, 430, 2327

Liu G., Zakamska N. L., Greene J. E., Nesvadba N., Liu X., 2013b, MNRAS, 436, 2576

Magorrian J. et al., 1998, AJ, 115, 2285

Martínez-Sansigre A., Rawlings S., Lacy M., Fadda D., Marleau F. R., Simpson C., Willott C. J., Jarvis M. J., 2005, Nature, 436, 666

Martínez-Sansigre A., Rawlings S., Lacy M., Fadda D., Jarvis M. J., Marleau F. R., Simpson C., Willott C. J., 2006a, MNRAS, 370, 1479

Martínez-Sansigre A., Rawlings S., Garn T., Green D. A., Alexander P., Klöckner H.-R., Riley J. M., 2006b, MNRAS, 373, L80

McElroy R., Croom S., Pracy M., Sharp R., Ho I. T., Medling A., 2015, MNRAS, 446. 2186

Norman C. et al., 2002, ApJ, 571, 218

Ohta K., Yamada T., Nakanishi K., Ogasaka Y., Kii T., Hayashida K., 1996, ApJ, 458, L57

Ptak A., Zakamska N. L., Strauss M. A., Krolik J. H., Heckman T. M., Schneider D. P., Brinkmann J., 2006, ApJ, 637, 147

Puchwein E., Springel V., 2013, MNRAS, 428, 2966

Ramos Almeida C., Tadhunter C. N., Inskip K. J., Morganti R., Holt J., Dicken D., 2011, MNRAS, 410, 1550

Ramos Almeida C. et al., 2012, MNRAS, 419, 687

Ramos Almeida C., Bessiere P. S., Tadhunter C. N., Inskip K. J., Morganti R., Dicken D., González-Serrano J. I., Holt J., 2013, MNRAS, 436, 997
Rees M. J., 1989, MNRAS, 239, 1P

Reyes R. et al., 2008, AJ, 136, 2373

Rodríguez M. I., Villar-Martín M., Emonts B., Humphrey A., Drouart G., García Burillo S., Pérez Torres M., 2014, A\&A, 565, A19

Sanders D. B., Soifer B. T., Elias J. H., Madore B. F., Matthews K., Neugebauer G., Scoville N. Z., 1988, ApJ, 325, 74

Silk J., 2013, ApJ, 772, 112

Stroe A., Sobral D., Röttgering H. J. A., van Weeren R. J., 2014, MNRAS, 438, 1377

Tadhunter C. et al., 2011, MNRAS, 412, 960

Villar-Martín M., Humphrey A., Martínez-Sansigre A., Pérez-Torres M., Binette L., Zhang X. G., 2008, MNRAS, 390, 218

Villar-Martín M., Tadhunter C., Pérez E., Humphrey A., Martínez-Sansigre A., Delgado R. G., Pérez-Torres M., 2010, MNRAS, 407, L6

Villar-Martín M., Tadhunter C., Humphrey A., Fraga Encina R., González Delgado R., Pérez Torres M., Martínez-Sansigre A., 2011a, MNRAS, 416, 262 (VM11a)

Villar-Martín M., Humphrey A., González Delgado R., Colina L., Arribas S., 2011b, MNRAS, 418, 2032 (VM11b)

Villar-Martín M., Cabrera Lavers A., Bessiere P., Tadhunter C., Rose M., de Breuck C., 2012, MNRAS, 423, 80

Villar-Martín M., Emonts B., Rodríguez M., Torres M. P., Drouart G., 2013a, MNRAS, 432, 2104

Villar-Martín M. et al., 2013b, MNRAS, 434, 978

Villar-Martín M., Emonts B., Humphrey A., Cabrera Lavers A., Binette L., 2014, MNRAS, 440, 3202

Villar-Martín M., Bellocchi E., Stern J., Tadhunter C., González Delgado R., 2015, MNRAS, 454, 439

White S. D. M., Frenk C. S., 1991, ApJ, 379, 52

Zakamska N. L. et al., 2003, AJ, 126, 2125

Zakamska N. L., Strauss M. A., Heckman T. M., Ivezić Ž., Krolik J. H., 2004, AJ, 128, 1002

Zakamska N. L. et al., 2006, AJ, 132, 1496

This paper has been typeset from a $\mathrm{T}_{\mathrm{E}} \mathrm{X} / \mathrm{LAT}_{\mathrm{E}} \mathrm{X}$ file prepared by the author. 